CERN-TH/99-408

Cavendish-HEP-99/18

hep-ph/9912399

\title{
Theoretical Aspects of Particle Production H $^{\prime}$
}

\author{
B.R. Webber \\ Theory Division, CERN, 1211 Geneva 23, Switzerland \\ and \\ Cavendish Laboratory, University of Cambridge, \\ Cambridge CB3 0HE, U.K.2
}

\section{Introduction}

In these lectures I shall describe some of the latest data on particle production in highenergy collisions and compare them with theoretical calculations and models based on QCD. The discussion will concentrate mainly on hadron distributions in jets, which are the manifestation at the hadronic level of hard (high-momentum-transfer) scattering of the partons (quarks and gluons) which are the fundamental fields of QCD.

In sect. 2, the connection between parton and hadron distributions is made more precise using the concept of fragmentation functions. I concentrate in particular on the region of small momentum fractions, where interesting characteristic features of QCD are manifest. Next, in sect. 3, the various available models for the conversion of partons into hadrons are reviewed. In sect. 1 , the predictions of theory and models are compared with experimental data. After that, sect. 5 focuses on new data that show clearly the differences between jets that originate from quark and gluon fragmentation.

Deep inelastic lepton scattering (DIS) at HERA is a copious source of jets; sect. 6 discusses new results on the properties of jets in the so-called current and target fragmentation regions.

In sect. 7 I discuss new data on the fragmentation of heavy $(b)$ quark jets into B mesons, and finally sect. 8 draws some brief conclusions.

Many of the topics mentioned here are discussed more fully in ref. [1]. In order to bring the discussion up to date, I have tried wherever possible to refer to the very latest experimental data. Therefore many of the references and figures concern preliminary data shown only at conferences, in particular at the International Europhysics Conference on High Energy Physics (EPS-HEP 99) held in Tampere, Finland, in July 1999. The cited contributed papers can be found through the EPS-HEP 99 web page [2] or, in the case of the large collaborations, through the collaboration pages [3]-[10].

\footnotetext{
${ }^{1}$ Lectures at International Summer School on Particle Production Spanning $\mathrm{MeV}$ and $\mathrm{TeV}$ Energies, Nijmegen, The Netherlands, August 1999.

${ }^{2}$ Permanent address.
} 


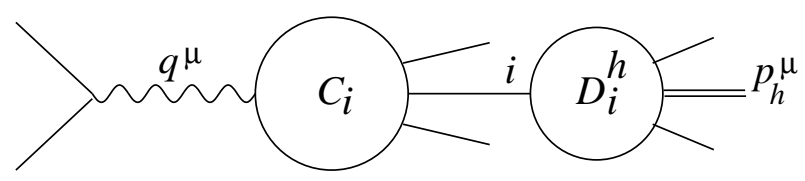

Figure 1: Factorization structure of $e^{+} e^{-} \rightarrow h X$.

\section{Jet fragmentation - theory}

We start with the basic factorization structure [11] of the single-particle inclusive distribution, e.g. in $e^{+} e^{-} \rightarrow h X$ (fig. [1):

$$
\begin{gathered}
F^{h}(x, s)=\sum_{i} \int_{x}^{1} \frac{d z}{z} C_{i}\left(z, \alpha_{\mathrm{S}}(s)\right) D_{i}^{h}(x / z, s), \\
s=q^{2}, \quad x=2 p_{h} \cdot q / q^{2}=2 E_{h} / E_{c m}
\end{gathered}
$$

where $C_{i}$ are the coefficient functions for this particular process (including all selection cuts etc.) and $D_{i}^{h}$ is the universal fragmentation function [12] for parton $i \rightarrow$ hadron $h$.

Although universal, fragmentation functions are factorization scheme dependent [11]. If one tries to calculate them in perturbation theory, one encounters divergences associated with the propagation of partons over long distances. In reality, however, partons are confined and cannot travel long distances. The perturbative divergences can be collected into overall factors that are replaced by non-perturbative factors taken from experiment. In this way the incorrect long-distance behaviour of perturbation theory is replaced by the correct long-distance features of QCD. However, the factorization of the divergent terms is ambiguous: one can choose to include different finite parts as well. This is the factorization scheme ambiguity. To specify the scheme requires calculation of the coefficient functions to (at least) next-to-leading order. This has only been done in a few cases. Thus there is need for theoretical work to make full use of the data on fragmentation functions.

In certain kinematic regions, higher-order corrections are enhanced by large logarithms, which need to be resummed to all orders. Large logarithms of ratios of invariants may appear inside the coefficient functions $C_{i}$, for example in multi-jet processes when the angles between jets become small. In some cases these can be absorbed into a change of scale in the fragmentation functions. Examples of this will be encountered in sects. 1 and 5 .

The fragmentation functions $D_{i}^{h}$ are not perturbatively calculable but their $s$-dependence (scaling violation) is given by the DGLAP evolution equation [13, 14, 15]:

$$
s \frac{\partial}{\partial s} D_{i}^{h}(x, s)=\sum_{j} \int_{x}^{1} \frac{d z}{z} P_{j i}\left(z, \alpha_{\mathrm{S}}(s)\right) D_{j}^{h}(x / z, s)
$$

where $P_{j i}$ is the parton $i \rightarrow j$ splitting function. Thus fragmentation functions can be parametrized at some fixed scale $s_{0}$ and then predicted at other energies [12].

The most common strategy for solving the DGLAP equation is to take moments (Mellin transforms) with respect to $x$ :

$$
\tilde{D}(N, s)=\int_{0}^{1} d x x^{N-1} D(x, s),
$$


the inverse Mellin transform being

$$
D(x, s)=\frac{1}{2 \pi i} \int_{C} d N x^{-N} \tilde{D}(N, s),
$$

where the integration contour $C$ in the complex $N$ plane is parallel to the imaginary axis and to the right of all singularities of the integrand. After Mellin transformation, the convolution on the right-hand side of eq. (3) becomes simply a product.

The moments $\tilde{P}_{j i}$ of the splitting functions are called anomalous dimensions, usually denoted by $\gamma_{j i}\left(N, \alpha_{\mathrm{S}}\right)$. They have perturbative expansions of the form

$$
\gamma_{j i}\left(N, \alpha_{\mathrm{S}}\right)=\sum_{n=0}^{\infty} \gamma_{j i}^{(n)}(N)\left(\frac{\alpha_{\mathrm{S}}}{2 \pi}\right)^{n+1}
$$

We can consider fragmentation function combinations which are non-singlet in flavour, such as $D_{V}=D_{q_{i}}-D_{\bar{q}_{i}}$ or $D_{q_{i}}-D_{q_{j}}$. In these combinations the mixing with the flavoursinglet gluon drops out and for a fixed value of $\alpha_{\mathrm{S}}$ the solution is simply

$$
\tilde{D}_{V}(N, s)=\tilde{D}_{V}\left(N, s_{0}\right)\left(\frac{s}{s_{0}}\right)^{\gamma_{q q}\left(N, \alpha_{S}\right)} .
$$

For a running coupling $\alpha_{\mathrm{S}}(s)$, the scaling violation is no longer power-behaved in $s$. The lowest-order form of the running coupling is

$$
\alpha_{\mathrm{S}}(s)=\frac{1}{b \ln \left(s / \Lambda^{2}\right)}
$$

with $b=\left(11 C_{A}-2 n_{f}\right) / 12 \pi$, where $C_{A}=3$ for QCD and $n_{f}$ is the number of 'active' quark flavours, i.e. the number with $m_{q}^{2} \ll s$. Using this we find the solution

$$
\tilde{D}_{V}(N, s)=\tilde{D}_{V}\left(N, s_{0}\right)\left(\frac{\alpha_{\mathrm{S}}\left(s_{0}\right)}{\alpha_{\mathrm{S}}(s)}\right)^{d_{q q}(N)}, d_{q q}(N)=\frac{\gamma_{q q}^{(0)}(N)}{2 \pi b},
$$

which varies like a power of $\ln s$.

For the singlet fragmentation function

$$
D_{S}=\sum_{i}\left(D_{q_{i}}+D_{\bar{q}_{i}}\right)
$$

we have mixing with the fragmentation of the gluon and the evolution equation becomes a matrix relation of the form

$$
s \frac{\partial}{\partial s}\left(\begin{array}{c}
D_{S} \\
D_{g}
\end{array}\right)=\left(\begin{array}{cc}
\gamma_{q q} & 2 n_{f} \gamma_{g q} \\
\gamma_{q g} & \gamma_{g g}
\end{array}\right)\left(\begin{array}{c}
D_{S} \\
D_{g}
\end{array}\right) .
$$

The anomalous dimension matrix in this equation has two real eigenvalues $\gamma_{ \pm}$given by

$$
\gamma_{ \pm}=\frac{1}{2}\left[\gamma_{g g}+\gamma_{q q} \pm \sqrt{\left(\gamma_{g g}-\gamma_{q q}\right)^{2}+8 n_{f} \gamma_{g q} \gamma_{q g}}\right]
$$

Expressing $D_{S}$ and $D_{g}$ as linear combinations of the corresponding eigenvectors $D_{+}$and $D_{-}$, we find that they evolve as superpositions of terms of the form (9) with $\gamma_{+}$and $\gamma_{-}$in the place of $\gamma_{q q}$. 
At small $x$, corresponding to $N \rightarrow 1$, the $q \rightarrow g$ and $g \rightarrow g$ anomalous dimensions have a singularity,

$$
\gamma_{g q} \rightarrow \frac{C_{F} \alpha_{\mathrm{S}}}{\pi(N-1)}+\mathcal{O}\left(\alpha_{\mathrm{S}}^{2}\right), \quad \gamma_{g g} \rightarrow \frac{C_{A} \alpha_{\mathrm{S}}}{\pi(N-1)}+\mathcal{O}\left(\alpha_{\mathrm{S}}^{2}\right)
$$

$\left(C_{F}=4 / 3\right)$, and we find $\gamma_{+} \rightarrow \gamma_{g g} \rightarrow \infty, \gamma_{-} \rightarrow \gamma_{q q} \rightarrow 0$. Thus the low- $x$ region requires special treatment, as we discuss in the following subsection.

\subsection{Small-x fragmentation}

At small $x$, multiple soft parton emission gives rise to terms enhanced by up to two powers of $\ln x$ for each power of $\alpha_{\mathrm{S}}$. The leading enhanced terms can be resummed by changing the DGLAP equation (3) to

$$
s \frac{\partial}{\partial s} D_{i}^{h}(x, s)=\sum_{j} \int_{x}^{1} \frac{d z}{z} P_{j i}\left(z, \alpha_{\mathrm{S}}(s)\right) D_{j}^{h}\left(x / z, z^{2} s\right) .
$$

The fact that the scale on the right-hand side should be $z^{2} s$ rather than $s$ follows from angular ordering of successive parton emissions [16, 17].

For simplicity, consider first the solution of eq. (14) for gluon fragmentation, taking $\alpha_{\mathrm{S}}$ fixed and neglecting the sum over different partons. Then taking moments as before we have

$$
s \frac{\partial}{\partial s} \tilde{D}(N, s)=\int_{0}^{1} d z z^{N-1} P\left(z, \alpha_{\mathrm{S}}\right) \tilde{D}\left(N, z^{2} s\right) .
$$

Now if we try a solution of the form

$$
D(N, s) \propto s^{\gamma\left(N, \alpha_{\mathrm{S}}\right)}
$$

we find that the anomalous dimension $\gamma\left(N, \alpha_{\mathrm{S}}\right)$ must satisfy the implicit equation

$$
\gamma\left(N, \alpha_{\mathrm{S}}\right)=\int_{0}^{1} d z z^{N-1+2 \gamma\left(N, \alpha_{\mathrm{S}}\right)} P\left(z, \alpha_{\mathrm{S}}\right) .
$$

When $N-1$ is not small, we can neglect the $2 \gamma\left(N, \alpha_{\mathrm{S}}\right)$ in the exponent of eq. (17) and then we obtain the usual explicit formula for the anomalous dimension. For $N \simeq 1$, the region we are interested in, the $z \rightarrow 0$ behaviour $P_{g g} \rightarrow C_{A} \alpha_{\mathrm{S}} / \pi z$ dominates, which implies that near $N=1$

$$
\gamma_{g g}\left(N, \alpha_{\mathrm{S}}\right)=\frac{C_{A} \alpha_{\mathrm{S}}}{\pi} \frac{1}{N-1+2 \gamma_{g g}\left(N, \alpha_{\mathrm{S}}\right)}
$$

and hence

$$
\begin{aligned}
& \gamma_{g g}\left(N, \alpha_{\mathrm{S}}\right)=\frac{1}{4}\left[\sqrt{(N-1)^{2}+\frac{8 C_{A} \alpha_{\mathrm{S}}}{\pi}}-(N-1)\right] \\
& =\sqrt{\frac{C_{A} \alpha_{\mathrm{S}}}{2 \pi}}-\frac{1}{4}(N-1)+\frac{1}{32} \sqrt{\frac{2 \pi}{C_{A} \alpha_{\mathrm{S}}}}(N-1)^{2}+\cdots
\end{aligned}
$$

Thus for $N \rightarrow 1$ the gluon-gluon anomalous dimension behaves like the square root of $\alpha_{\mathrm{S}}$. How can this behaviour emerge from perturbation theory, which deals only in integer 
powers of $\alpha_{\mathrm{S}}$ ? The answer is that at any fixed $N \neq 1$ we can expand eq. (19) in a different way for sufficiently small $\alpha_{\mathrm{S}}$ :

$$
\gamma_{g g}\left(N, \alpha_{\mathrm{S}}\right)=\frac{C_{A} \alpha_{\mathrm{S}}}{\pi(N-1)}-2\left(\frac{C_{A} \alpha_{\mathrm{S}}}{\pi}\right)^{2} \frac{1}{(N-1)^{3}}+\cdots
$$

This series displays the terms that are most singular as $N \rightarrow 1$ in each order. These terms have been resummed in the expression (19), allowing the perturbation series to be analytically continued outside its circle of convergence $\left|\alpha_{S}\right|<\left(\pi / 8 C_{A}\right)|N-1|^{2}$. By definition, the behaviour outside this circle (in particular, at $N=1$ ) cannot be represented by the series any more, even though it is fully implied by it.

At sufficiently small $x$, the $N \rightarrow 1$ singularity of the gluon-gluon anomalous dimension dominates in all fragmentation functions, and this in turn determines the asymptotic behaviour of the single-particle inclusive distributions $F^{h}$ in eq. (看). To predict this behaviour quantitatively we need to take account of the running of $\alpha_{\mathrm{S}}$, which can be done by writing eq. (16) in the form

$$
\tilde{D}(N, s) \sim \exp \left[\int^{s} \gamma_{g g}\left(N, \alpha_{\mathrm{S}}\right) \frac{d s^{\prime}}{s^{\prime}}\right]
$$

and noting that $\alpha_{\mathrm{S}}$ in the integrand should be $\alpha_{\mathrm{S}}\left(s^{\prime}\right)$. We then use eq. (8) to write

$$
\int^{s} \gamma_{g g}\left(N, \alpha_{\mathrm{S}}\left(s^{\prime}\right)\right) \frac{d s^{\prime}}{s^{\prime}}=-\frac{1}{b} \int^{\alpha_{\mathrm{S}}(s)} \gamma_{g g}\left(N, \alpha_{\mathrm{S}}\right) \frac{d \alpha_{\mathrm{S}}}{\alpha_{\mathrm{S}}^{2}}
$$

and hence

$$
\begin{aligned}
\tilde{D}(N, s) & \propto \exp \left[\frac{1}{b} \sqrt{\frac{2 C_{A}}{\pi \alpha_{\mathrm{S}}}}-\frac{1}{4 b \alpha_{\mathrm{S}}}(N-1)\right. \\
& \left.+\frac{1}{48 b} \sqrt{\frac{2 \pi}{C_{A} \alpha_{\mathrm{S}}^{3}}}(N-1)^{2}+\cdots\right]_{\alpha_{\mathrm{S}}=\alpha_{\mathrm{S}}(s)} .
\end{aligned}
$$

The value of $\tilde{D}(N, s)$ at $N=1$ is simply the integral of the fragmentation function, which gives the average multiplicity,

$$
N_{i}^{h}(s) \sim \tilde{D}_{i}^{h}(1, s) \propto \exp \left[\frac{1}{b} \sqrt{\frac{2 C_{A}}{\pi \alpha_{\mathrm{S}}(s)}}\right] \sim \exp \sqrt{\frac{2 C_{A}}{\pi b} \ln s} .
$$

Thus the average multiplicity of any hadron species should increase asymptotically faster than any power of $\ln s$ but slower than any positive power of $s$. Furthermore the relations (13) imply that the average multiplicities in gluon and quark jets are asymptotically in the ratio of their 'colour charges' $C_{A}$ and $C_{F}$ [18]:

$$
\frac{N_{g}^{h}(s)}{N_{q}^{h}(s)} \rightarrow \frac{C_{A}}{C_{F}}=\frac{9}{4}
$$

The behaviour of $\tilde{D}(N, s)$ near $N=1$ determines the form of small- $x$ fragmentation functions via the inverse Mellin transformation (5). Keeping the first three terms in the 
Taylor expansion of the exponent, as displayed in eq. (23), gives a simple Gaussian function of $N$ which transforms into a Gaussian in the variable $\xi \equiv \ln (1 / x)$ :

$$
x D(x, s) \propto \exp \left[-\frac{1}{2 \sigma^{2}}\left(\xi-\xi_{p}\right)^{2}\right]
$$

where the peak position is

$$
\xi_{p}=\frac{1}{4 b \alpha_{\mathrm{S}}(s)} \sim \frac{1}{4} \ln s
$$

and the width of the distribution of $\xi$ is

$$
\sigma=\left(\frac{1}{24 b} \sqrt{\frac{2 \pi}{C_{A} \alpha_{\mathrm{S}}^{3}(s)}}\right)^{\frac{1}{2}} \propto(\ln s)^{\frac{3}{4}} .
$$

Thus the effect of resummation is to generate a characteristic hump-backed shape in the variable $\xi=\ln (1 / x)$, with a peak that moves up and expands slowly with increasing $s$. Including also next-to-leading logarithms, one obtains what is commonly known as the modified leading-logarithmic approximation (MLLA) [19, 20, 21].

\section{Hadronization Models}

\subsection{General ideas}

Before discussing specific models for the hadronization process, we should review some general ideas that have proved useful in interpreting hadronization data.

- Local parton-hadron duality [22]. Hadronization is long-distance process, involving only small momentum transfers. Hence the flows of energy-momentum and flavour quantum numbers at hadron level should follow those at parton level. Results on inclusive spectra and multiplicities support this hypothesis.

- Universal low-scale $\alpha_{S}$ [23, 24, 25]. Perturbation theory works well down to low scales, $Q \sim 1 \mathrm{GeV}$. Assume therefore that $\alpha_{\mathrm{S}}\left(Q^{2}\right)$ can be defined non-perturbatively for all $Q$, and use it in evaluation of Feynman graphs. This approach gives a good description of heavy quark spectra and event shapes.

\subsection{Specific models}

The above general ideas do not try to describe the mechanism of hadron formation. For this we must resort to models. The main current models are cluster and string hadronization. We describe briefly the versions used in the HERWIG and JETSET Monte Carlo event generators, respectively. In both cases, a parton shower initiated by the hard process evolves

perturbatively, according to the DGLAP equation, until the scale of parton virtualities has fallen to some low value $Q_{0} \sim 1 \mathrm{GeV}$, whereupon the non-perturbative processes assumed in the model take over (fig. 2). 


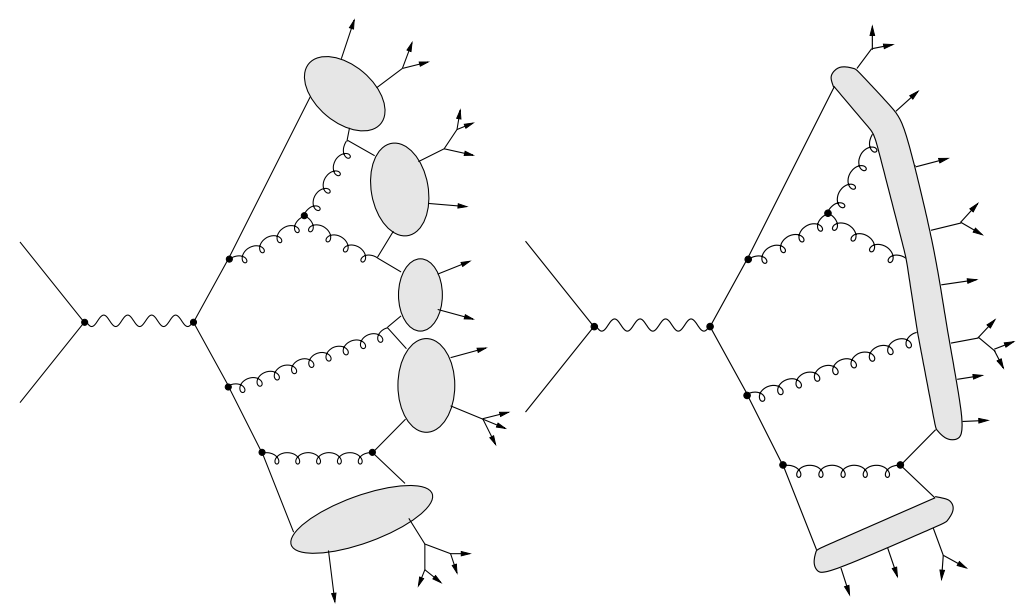

Figure 2: Cluster and string hadronization models.

- Cluster model [26]-[29]. The model starts with non-perturbative splitting of all gluons after the parton shower, $g \rightarrow q \bar{q}$. Colour-singlet $q \bar{q}$ combinations have lower masses and a universal spectrum due to the preconfinement [30, 31] property of the shower (fig. 3 [32]). These colour-singlet combinations are assumed to form clusters, which mostly undergo simple isotropic decay into pairs of hadrons, chosen according to the density of states with appropriate quantum numbers [27]. This model has few parameters and a natural mechanism for generating transverse momenta and suppressing heavy particle production in hadronization. However, it has problems in dealing with the decay of very massive clusters, and in adequately suppressing baryon and heavy quark production.

- String model [33-36]. This model is based on the dynamics of a relativistic string, representing the colour flux stretched between the initial $q \bar{q}$. The string produces a linear confinement potential and an area law for matrix elements:

$$
\left|M\left(q \bar{q} \rightarrow h_{1} \cdots h_{n}\right)\right|^{2} \propto e^{-b A}
$$

where $A$ is the space-time area swept out (fig. (4). The string breaks up into hadrons via $q \bar{q}$ pair production in its intense colour field. Gluons produced in the parton shower give rise to 'kinks' on the string. The model has extra parameters for the transverse momentum distribution and heavy particle suppression. It has some problems describing baryon production, but less than the cluster model.

- The UCLA model [37, 38] is a variant of the JETSET string model which takes the above area law for matrix elements more seriously, using it to determine the relative rates of production of different hadron species. This results in heavy particle suppression without extra parameters, the mass-squared of a hadron being proportional to its space-time area. At present the model still uses extra parameters for $p_{T}$ spectra, and again has some problems describing baryon production. 


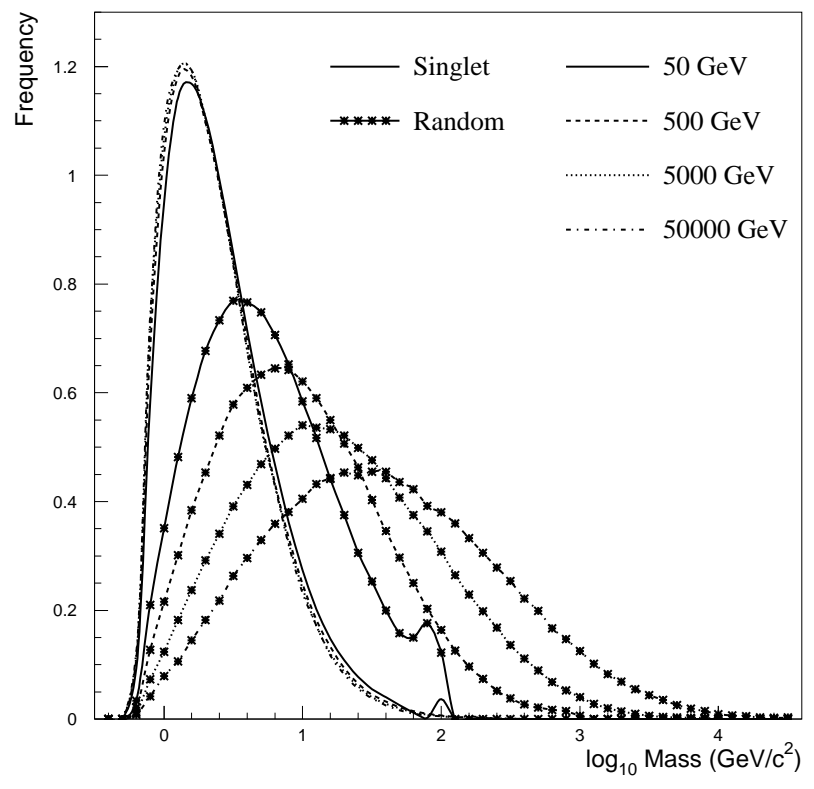

Figure 3: Cluster model: mass distribution of $q \bar{q}$ pairs.

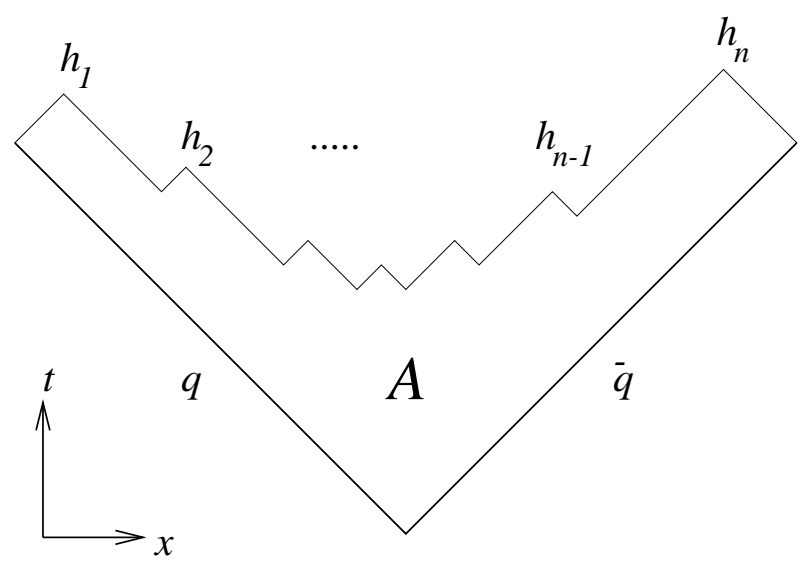

Figure 4: String model: space-time picture. 


\begin{tabular}{|c|c|c|c|c|c|}
\hline Particle & Multiplicity & HERWIG & JETSET & UCLA & Expts \\
& & 5.9 & 7.4 & 7.4 & \\
\hline Charged & $20.96(18)$ & 20.95 & 20.95 & 20.88 & ADLMO \\
$\pi^{ \pm}$ & $17.06(24)$ & 17.41 & 16.95 & 17.04 & ADO \\
$\pi^{0}$ & $9.43(38)$ & 9.97 & 9.59 & 9.61 & ADLO \\
$\boldsymbol{\eta}$ & $0.99(4)$ & 1.02 & 1.00 & $\underline{0.78}$ & ALO \\
$\rho(770)^{0}$ & $1.24(10)$ & 1.18 & 1.50 & 1.17 & AD \\
$\omega(782)$ & $1.09(9)$ & 1.17 & 1.35 & 1.01 & ALO \\
$\boldsymbol{\eta}^{\prime}(\mathbf{9 5 8})$ & $0.159(26)$ & 0.097 & 0.155 & 0.121 & ALO \\
$\mathrm{f}_{\mathbf{0}}(\mathbf{9 8 0})$ & $0.155(8)$ & $\underline{0.111}$ & $\sim \underline{0.1}$ & - & ADO \\
$\mathrm{a}_{0}(980)^{ \pm}$ & $0.14(6)$ & 0.240 & - & - & $\mathrm{O}$ \\
$\phi(1020)$ & $0.097(7)$ & 0.104 & $\underline{0.194}$ & $\underline{0.132}$ & $\mathrm{ADO}$ \\
$\mathrm{f}_{\mathbf{2}}(\mathbf{1 2 7 0})$ & $0.188(14)$ & 0.186 & $\sim 0.2$ & - & $\mathrm{ADO}$ \\
$\mathrm{f}_{2}^{\prime}(1525)$ & $0.012(6)$ & 0.021 & - & - & $\mathrm{D}$ \\
\hline $\mathrm{K}^{ \pm}$ & $2.26(6)$ & 2.16 & 2.30 & 2.24 & $\mathrm{ADO}$ \\
$\mathrm{K}^{\mathbf{0}}$ & $2.074(14)$ & 2.05 & 2.07 & 2.06 & $\mathrm{ADLO}$ \\
$\mathrm{K}^{*}(892)^{ \pm}$ & $0.718(44)$ & 0.670 & $\underline{1.10}$ & 0.779 & $\mathrm{ADO}$ \\
$\mathrm{K}^{*}(892)^{0}$ & $0.759(32)$ & 0.676 & $\underline{1.10}$ & 0.760 & $\mathrm{ADO}$ \\
$\mathrm{K}_{2}^{*}(1430)^{0}$ & $0.084(40)$ & 0.111 & - & - & $\mathrm{DO}$ \\
\hline $\mathrm{D}^{ \pm}$ & $0.187(14)$ & $\underline{0.276}$ & 0.174 & 0.196 & $\mathrm{ADO}$ \\
$\mathrm{D}^{0}$ & $0.462(26)$ & 0.506 & 0.490 & 0.497 & $\mathrm{ADO}$ \\
$\mathrm{D}^{*}(2010)^{ \pm}$ & $0.181(10)$ & 0.161 & $\underline{0.242}$ & $\underline{0.227}$ & $\mathrm{ADO}$ \\
$\mathrm{D}_{\mathrm{s}}^{ \pm}$ & $0.131(20)$ & 0.115 & 0.129 & 0.130 & $\mathrm{O}$ \\
\hline $\mathrm{B}^{*}$ & $0.28(3)$ & 0.201 & 0.260 & 0.254 & $\mathrm{D}$ \\
$\mathrm{B}_{\mathrm{u}, \mathrm{d}}^{* *}$ & $0.118(24)$ & $\underline{0.013}$ & - & - & $\mathrm{D}$ \\
\hline $\mathrm{J}^{\mathbf{4}} \boldsymbol{\psi}$ & $0.0054(4)$ & $\underline{0.0018}$ & 0.0050 & 0.0050 & $\mathrm{ADLO}$ \\
$\boldsymbol{\chi}_{(3685)}$ & $0.0023(5)$ & 0.0009 & 0.0019 & 0.0019 & $\mathrm{DO}$ \\
$\chi_{\mathrm{c} 1}$ & $0.0086(27)$ & $\underline{0.0001}$ & - & - & $\mathrm{DL}$ \\
\hline
\end{tabular}

Table 1: Meson yields in $\mathrm{Z}^{0}$ decay. Experiments: $\mathrm{A}=\mathrm{Aleph}, \mathrm{D}=$ Delphi, $\mathrm{L}=\mathrm{L} 3, \mathrm{M}=\mathrm{Mark}$ II, $\mathrm{O}=$ Opal. Bold: new data this year. Underlined: disagreement with data by more than $3 \sigma$.

\section{Single-particle yields and spectra}

Tables 1 and $2^{3}$ compare predictions of the above models with data on $\mathrm{Z}^{0}$ decay from LEP and SLC. Of course, the models have tunable parameters, but the overall agreement is encouraging. As stated earlier, the main problems are in the baryon sector, especially for HERWIG.

It is remarkable that most measured yields (except for the $0^{-}$mesons, which have special status as Goldstone bosons) lie on the family of curves

$$
\langle n\rangle=a(2 J+1) e^{-M / T}
$$

where $M$ is the mass and $T \simeq 100 \mathrm{MeV}$ (fig. 5 [40]). This suggests that mass, rather than quantum numbers, is the primary factor in determining production rates. Note that,

\footnotetext{
${ }^{3}$ Updated from ref. [32].

${ }^{4}$ Recent ALEPH HERWIG tuning with strangeness suppression 0.8 [39].
} 


\begin{tabular}{|c|c|c|c|c|c|}
\hline Particle & Multiplicity & $\begin{array}{c}\text { HERWIG } \\
5.9\end{array}$ & $\begin{array}{c}\text { JETSET } \\
7.4\end{array}$ & $\begin{array}{c}\text { UCLA } \\
7.4\end{array}$ & Expts \\
\hline $\mathrm{p}$ & $1.04(4)$ & $\underline{0.863}$ & $\underline{1.19}$ & 1.09 & ADO \\
\hline$\Delta^{++}$ & $0.079(15)$ & $\underline{0.156}$ & $\underline{0.189}$ & $\underline{0.139}$ & $\mathrm{D}$ \\
& $0.22(6)$ & 0.156 & 0.189 & 0.139 & O \\
\hline $\boldsymbol{\Lambda}$ & $0.399(8)$ & 0.387 & 0.385 & 0.382 & ADLO \\
$\boldsymbol{\Lambda}(\mathbf{1 5 2 0})$ & $0.0229(25)$ & - & - & - & DO \\
\hline$\Sigma^{ \pm}$ & $0.174(16)$ & 0.154 & 0.140 & 0.118 & DO \\
$\Sigma^{0}$ & $0.074(9)$ & 0.068 & 0.073 & 0.074 & ADO \\
$\Sigma^{\star \pm}$ & $0.0474(44)$ & $\underline{0.111}$ & $\underline{0.074}$ & $\underline{0.074}$ & ADO \\
\hline$\Xi^{-}$ & $0.0265(9)$ & $\underline{0.0493}$ & 0.0271 & $\underline{0.0220}$ & ADO \\
$\Xi(1530)^{0}$ & $0.0058(10)$ & $\underline{0.0205}$ & 0.0053 & 0.0081 & ADO \\
\hline$\Omega^{-}$ & $0.0012(2)$ & $\underline{0.0056}$ & 0.00072 & 0.0011 & ADO \\
\hline$\Lambda_{\mathrm{c}}^{+}$ & $0.078(17)$ & $\underline{0.0123}$ & 0.059 & $\underline{0.026}$ & O \\
\hline
\end{tabular}

Table 2: Baryon yields in $\mathrm{Z}^{0}$ decay. Legend as in table 1.

surprisingly, the orbitally-excited $J=\frac{3}{2}$ baryon $\Lambda(1520)$ (not yet included in models) is produced almost as much as the unexcited $J=\frac{3}{2}$ baryon $\Sigma(1385)$ 41, 42.

At other energies, model predictions for identified particle yields are in broad agreement with $e^{+} e^{-}$data (fig. [43]), but statistics are of course poorer. Charged particle spectra at low $x$ agree well with the resummed (MLLA) predictions discussed in sect. 2 over a wide energy range, as illustrated in fig. 74 . 44 .

In $p \bar{p} \rightarrow$ dijets 45 the relevant scale is taken to be $Q=M_{J J} \sin \theta$ where $M_{J J}$ is the dijet mass and $\theta$ is the jet cone angle (fig. 8). Results are then in striking agreement with MLLA predictions and with data from $e^{+} e^{-}$annihilation at $Q=\sqrt{s}$ (fig. 9).

New SLD data include hadron spectra in light quark (rather than antiquark) fragmentation, selected by hemisphere using the SLC beam polarization [46]. One sees strong particle/antiparticle differences in the expected directions (fig. 10), bearing in mind the predominance of down-type quarks in $\mathrm{Z}^{0}$ decay.

\section{$5 \quad$ Quark and gluon jets}

DELPHI 47 select gluon jets by anti-tagging heavy quark jets in ' $\mathrm{Y}$ ' and 'Mercedes' three-jet events (fig. 11). As expected, the higher colour charge of the gluon $\left(C_{A}=3\right.$ vs. $C_{F}=4 / 3$ ) leads to a softer spectrum and higher overall multiplicity (fig. 12). In general the relative multiplicities of identified particles are consistent with those of all charged, with no clear excess of any species in gluon jets (fig. 13). In particular there is no enhanced $\phi(1020)$ or $\eta$ production:

DELPHI [47]: $N_{g}(\phi) / N_{q}(\phi)=0.7 \pm 0.3$

OPAL 48]: $N_{g}(\eta) / N_{q}(\eta)=1.29 \pm 0.11$

OPAL 49] select gluon jets recoiling against two tagged $b$-jets in the same hemisphere. Monte Carlo studies indicate that such jets should be similar to those emitted by a point source of gluon pairs. The qualitative message from the data is again clear (fig. 14): Gluon 


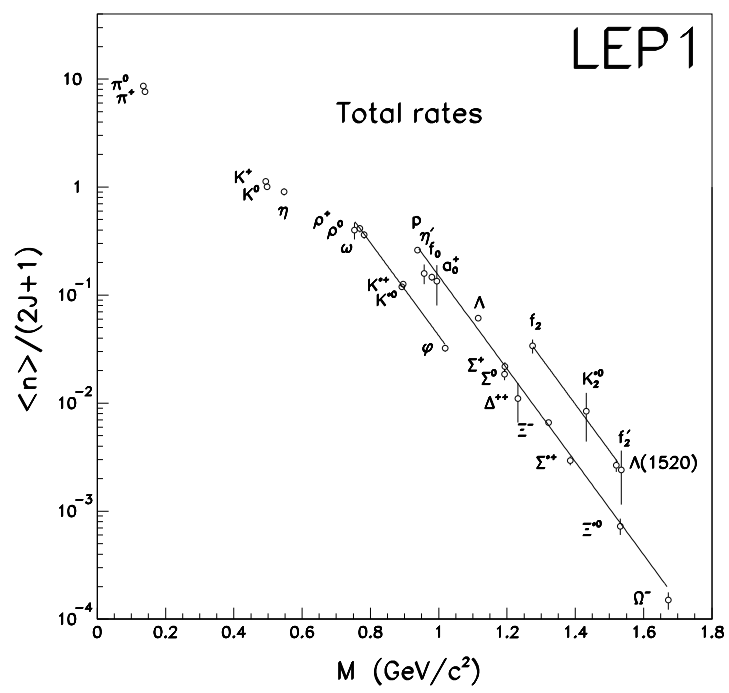

Figure 5: Particle yields in $Z^{0}$ decay.
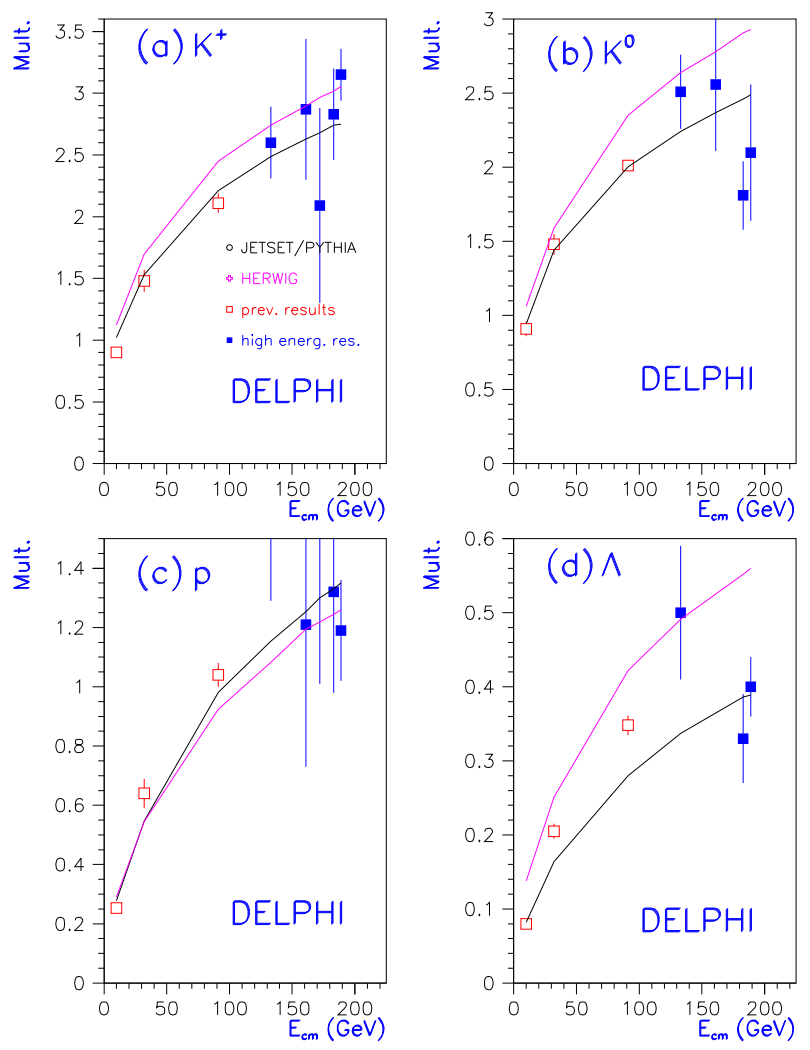

Figure 6: Particle yields in $e^{+} e^{-}$annihilation. 

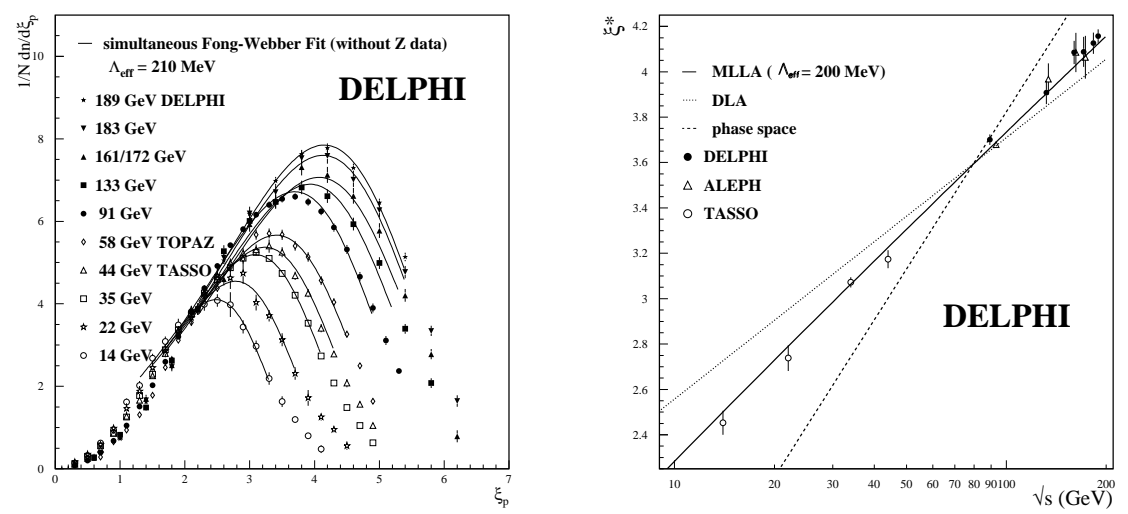

Figure 7: Low- $x$ fragmentation in $e^{+} e^{-}$annihilation.

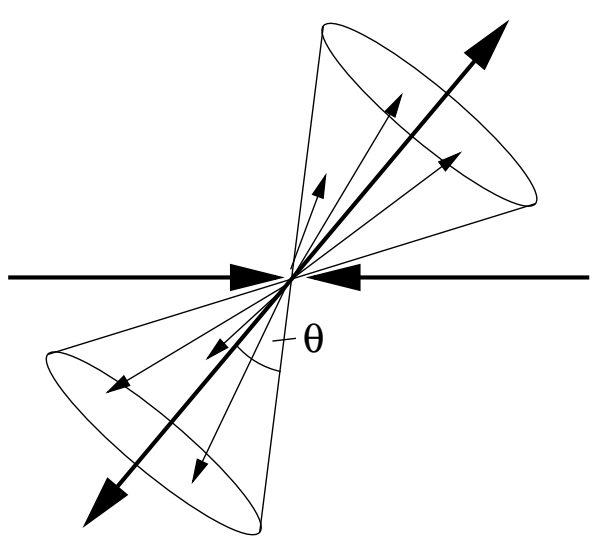

Figure 8: Cone angle in $p \bar{p} \rightarrow$ dijets.
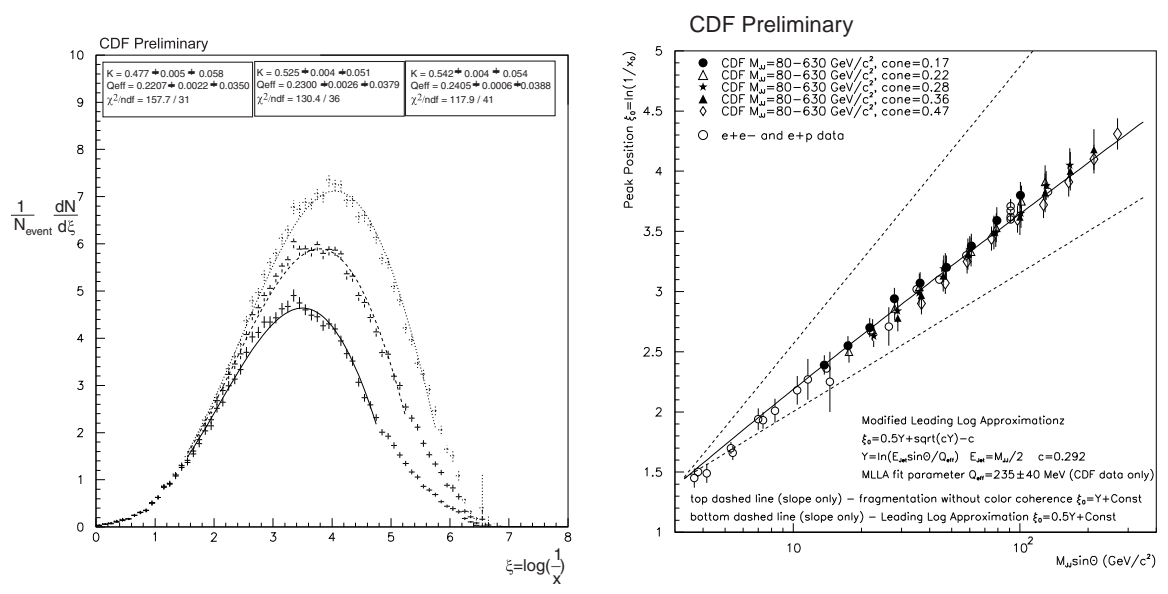

Figure 9: Low- $x$ fragmentation in $p \bar{p} \rightarrow$ dijets. 


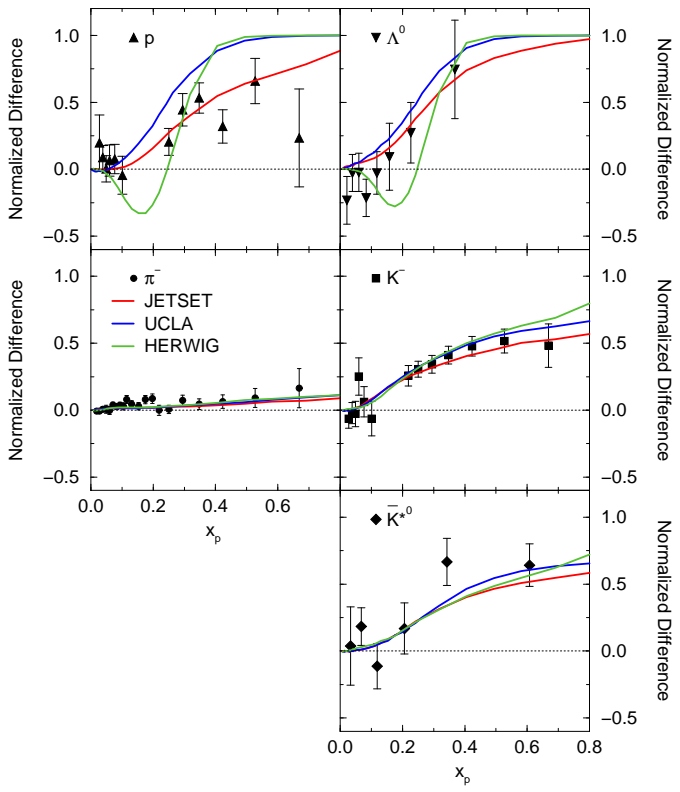

Figure 10: Normalized particle-antiparticle differences in quark jet fragmentation.

a)

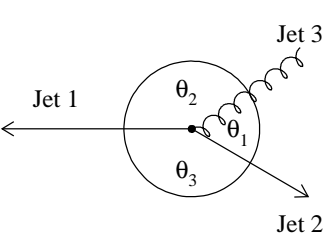

Y events b)

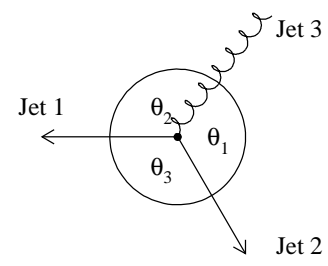

Mercedes events

Figure 11: Selection of gluon jets by DELPHI. 


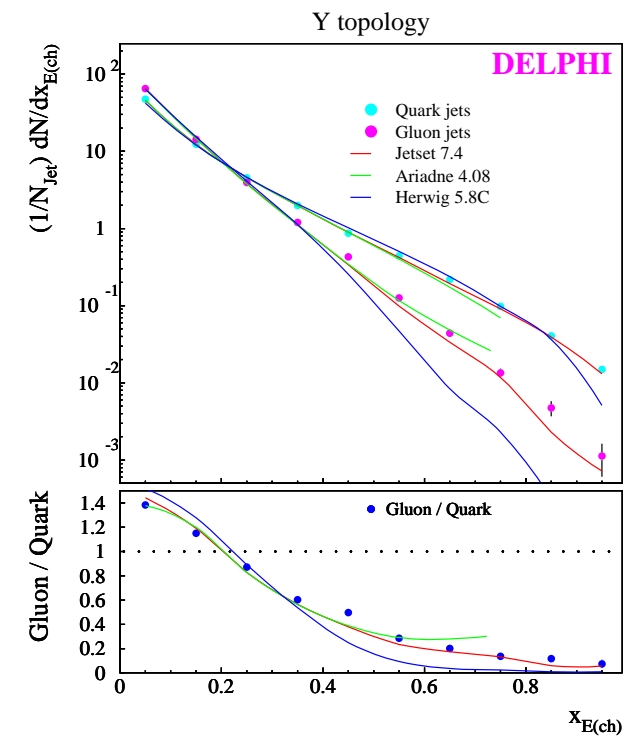

Figure 12: Charged particle spectra in quark and gluon jets.

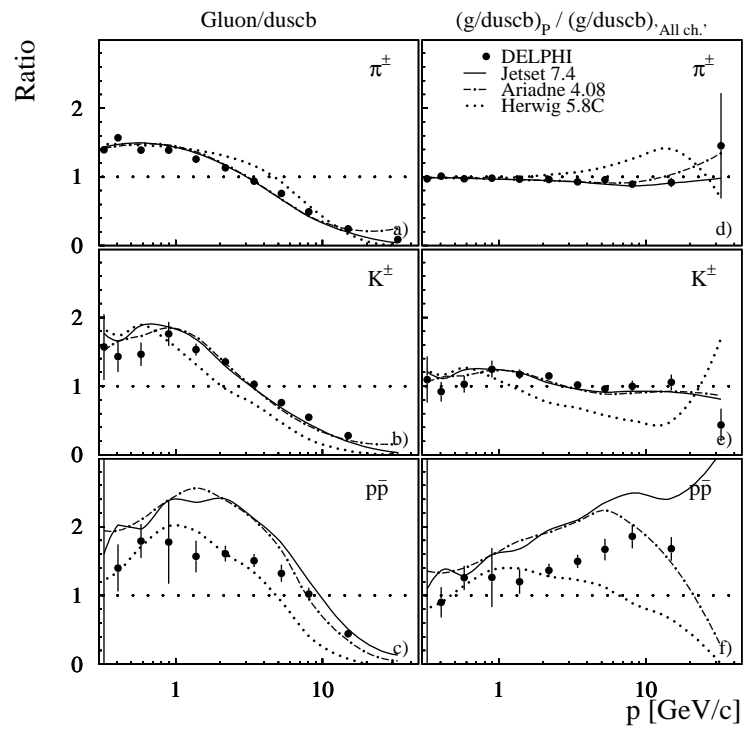

Figure 13: Comparisons of particle spectra in quark and gluon jets. 

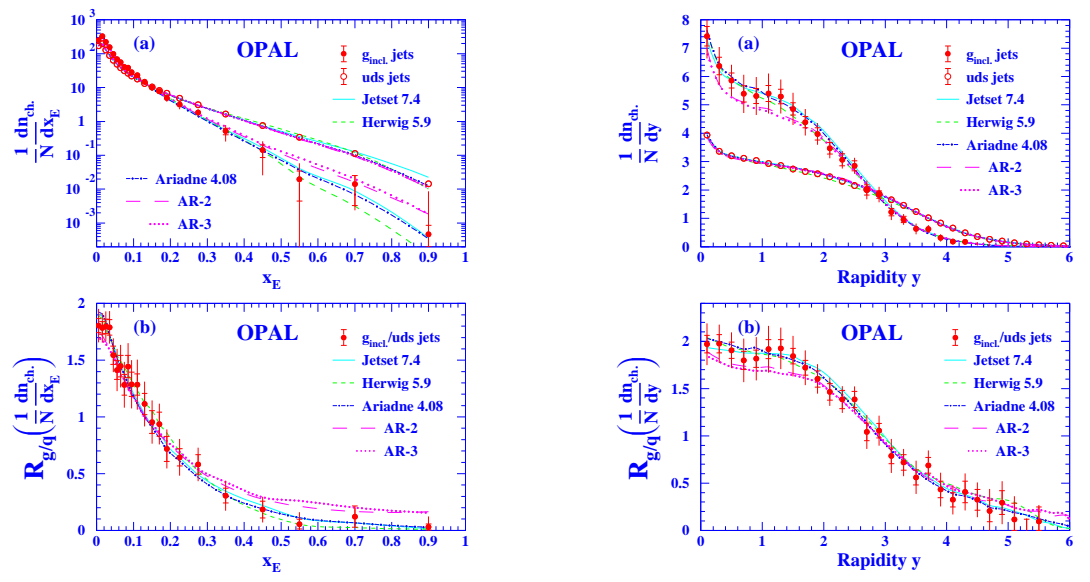

Figure 14: Momentum fraction and rapidity distributions in quark and gluon jets.

jets have softer fragmentation than light quark jets, and higher multiplicity. The precision of the data is now such that next-to-leading order calculations of the relevant coefficient functions, taking into account the experimental selection procedures, are needed to check universality of the extracted gluon fragmentation function.

The ratio of gluon/quark multiplicities at low rapidity (large angle) is close to the ratio of colour charges $r \equiv C_{A} / C_{F}=2.25$, in agreement with local parton-hadron duality:

$$
\text { OPAL: } r_{c h}(|y|<1)=1.919 \pm 0.047 \pm 0.095 \text {. }
$$

According to eq. (25), the overall multiplicity ratio should also approach the value $C_{A} / C_{F}$ asymptotically, but at present energies the contribution from higher rapidities is substantial and this leads to a smaller ratio.

Monte Carlo studies [49] suggest that a better measure of $C_{A} / C_{F}$ is obtained by selecting low-momentum hadrons with relatively large transverse momentum (i.e. low rapidity). This gives

$$
\text { OPAL: } r_{c h}\left(p<4,0.8<p_{T}<3 \mathrm{GeV}\right)=2.29 \pm 0.09 \pm 0.015 .
$$

DELPHI [50] have observed scaling violation in quark and gluon jet fragmentation separately (fig. 15) by studying the dependence on the scale

$$
\kappa_{H}=E_{\text {jet }} \sin (\theta / 2) \simeq \frac{1}{2} \sqrt{s y_{3}}
$$

where $\theta$ is the angle to the closest jet and $y_{3}$ is the Durham jet resolution [51] at which 3 jets are just resolved. This is expected to be the relevant scale when $y_{3}$ becomes small. One sees clearly that there is more scaling violation in gluon jets (fig. 16). The ratio provides another measure of $C_{A} / C_{F}$ :

$$
\text { DELPHI: } r_{\text {sc.viol. }}=2.23 \pm 0.09 \pm 0.06
$$

A crucial point in the DELPHI analysis is that 3-jet events are not selected using a fixed jet resolution $y_{\text {cut }}$, but rather each event is clustered to precisely 3 jets. This avoids 'biasing' the gluon jet sample by preventing further jet emission above $y_{\text {cut }}$. 

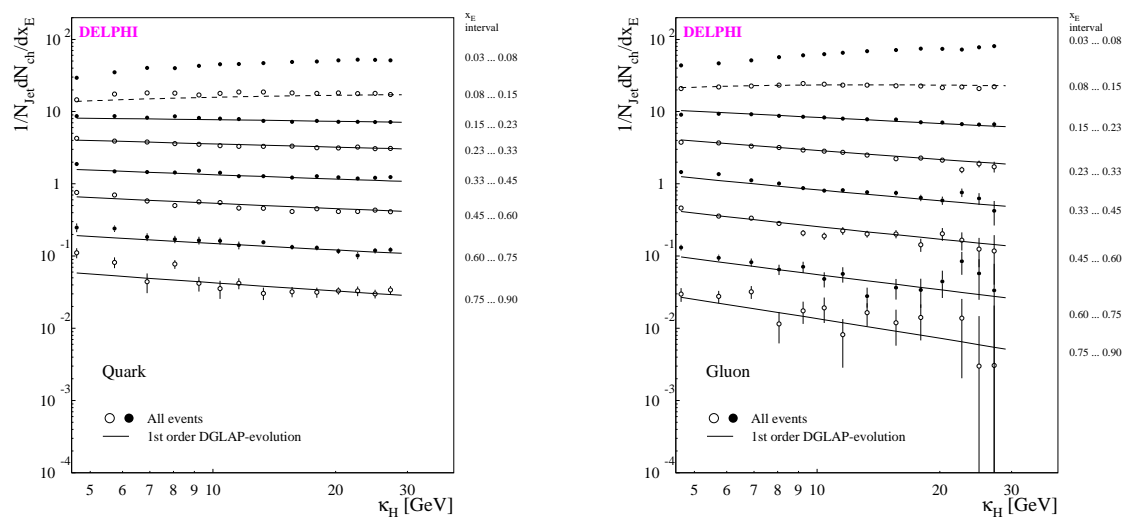

Figure 15: Scale dependence of quark and gluon fragmentation.

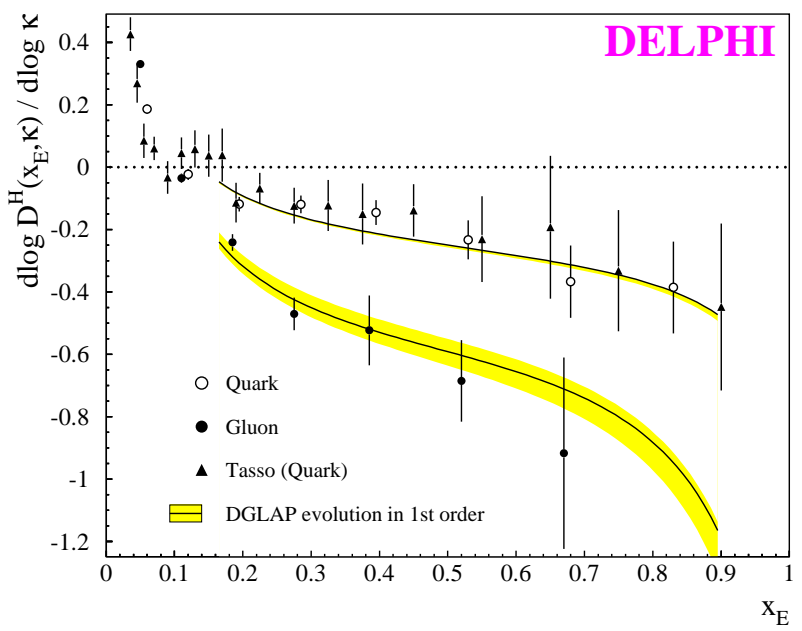

Figure 16: Logarithmic gradients of quark and gluon fragmentation. 


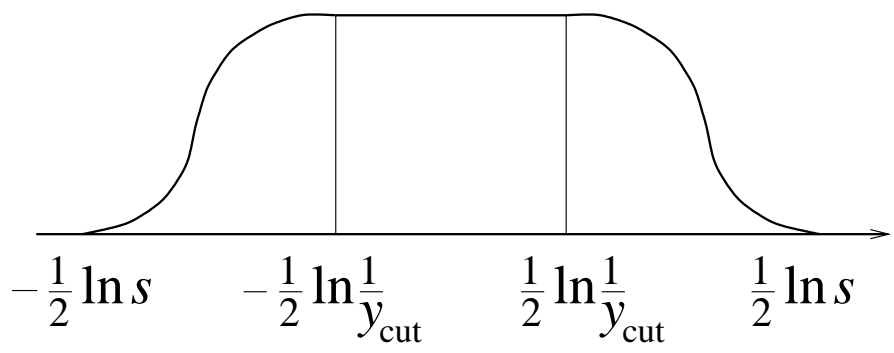

Figure 17: Rapidity plateau in two-jet events.

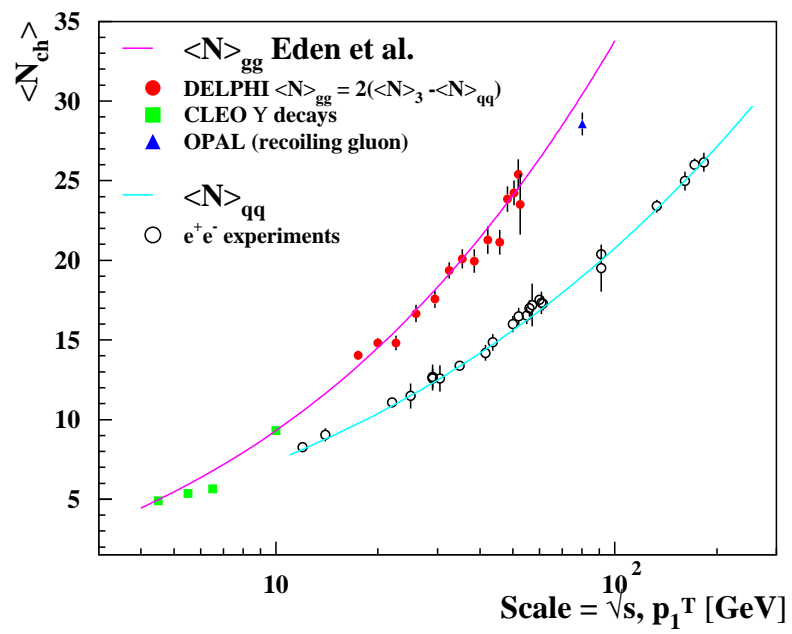

Figure 18: Average $q \bar{q}$ and $g g$ multiplicities deduced from 2- and 3-jet events.

The same point is well illustrated in analyses of average multiplicities in 2- and 3-jet events [52, 53, 54]. If $N_{q \bar{q}}(s)$ is the 'unbiased' $q \bar{q}$ multiplicity, then in events with precisely 2 jets at resolution $y_{\text {cut }}$ there is a rapidity plateau of length $\ln \left(1 / y_{\text {cut }}\right.$ ) (see fig. 17) and the multiplicity is

$$
N_{2}\left(s, y_{\text {cut }}\right) \simeq N_{q \bar{q}}\left(s y_{\text {cut }}\right)+\ln \left(1 / y_{\text {cut }}\right) N_{q \bar{q}}^{\prime}\left(s y_{\text {cut }}\right)
$$

where $N^{\prime}(s) \equiv s d N / d s$. Clustering each event to 3 jets we get this multiplicity with $y_{3}$ in place of $y_{\text {cut }}$, plus an unbiased gluon jet:

$$
N_{3}(s) \simeq N_{2}\left(s, y_{3}\right)+\frac{1}{2} N_{g g}\left(s y_{3}\right) .
$$

Thus one can extract the unbiased $g g$ multiplicity, plotted in fig. 18 vs. $p_{1}^{T} \sim \sqrt{s y_{3}}$ [55]. The ratio of $g g / q \bar{q}$ slopes gives yet another measure of $C_{A} / C_{F}$ [54]:

$$
r_{\text {mult }}=2.246 \pm 0.062 \text { (stat.) } \pm 0.080 \text { (sys.) } \pm 0.095 \text { (theo.) } .
$$

\section{Current and target fragmentation in DIS}

The H1 [56] and ZEUS [57] experiments at HERA have studied the distributions of $x_{p}=$ $2|\boldsymbol{p}| / Q$ in the current and target hemispheres in the Breit frame of reference. The Breit 


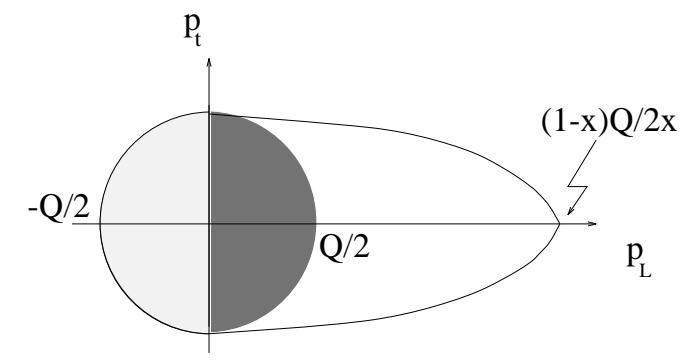

CURRENT TARGET

Figure 19: Breit frame current and target regions in DIS.

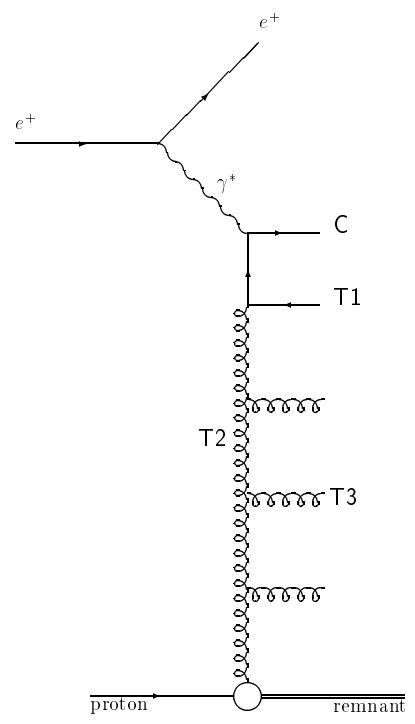

Figure 20: Contributions to the final state in DIS.

frame is the one in which the 4-momentum of the virtual photon exchanged in DIS lies entirely along the negative $z$-axis, $q^{\mu}=(0,0,0,-Q)$, while the target proton has $P^{\mu}=$ $(Q, 0,0, Q) / 2 x$ (neglecting the proton mass), where $x=-q^{2} / 2 p \cdot q$ is the Bjorken variable. In this frame, to zeroth order in $\alpha_{\mathrm{S}}$, the virtual photon simply strikes a constituent of the target with momentum $Q / 2$ and reverses its momentum. The remnant of the target is then left with momentum $(1-x) Q / 2 x$ (fig. 19).

In higher orders one expects the current hemisphere to contain fragmentation products of the current jet ( $\mathrm{C}$ in fig. 20), similar to half an $e^{+} e^{-}$event. In the target hemisphere, the contribution T1 is similar to $\mathrm{C}$, T2 gives extra particles with $x_{p}<1$, while T3 gives $x_{p} \gtrsim 1$, generally outside detector acceptance.

- In the current hemisphere the charged multiplicity is indeed similar to $e^{+} e^{-}$(fig. 21 [57]). Differences at low $Q^{2}$ are consistent with the expected boson-gluon fusion contribution. The distribution of $\xi=\ln \left(1 / x_{p}\right)$ is also similar to $e^{+} e^{-}$, i.e. close to Gaussian with little Bjorken $x$ dependence (fig. 22).

At low $Q^{2}$ there is evidence of strong subleading corrections. The distribution is skewed towards higher values of $\xi$ (smaller $x_{p}$ ), contrary to MLLA predictions (fig. 23). 


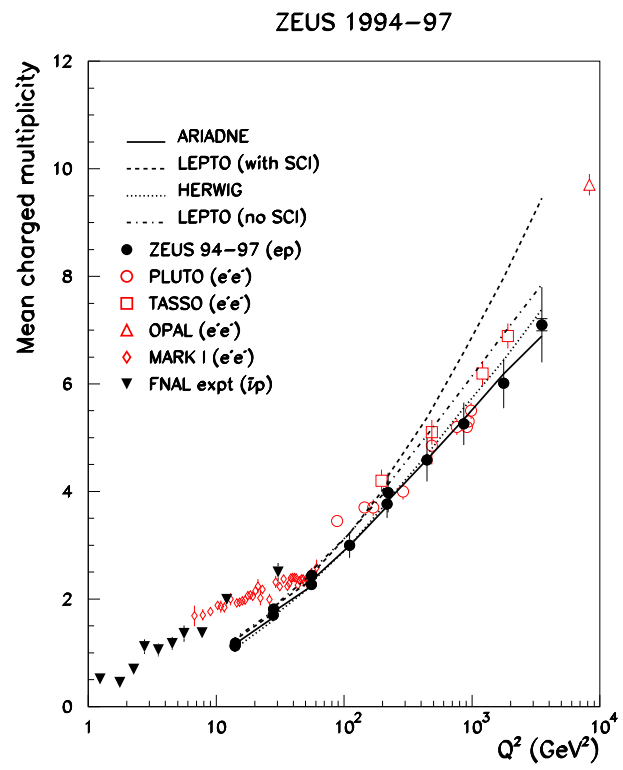

Figure 21: Charged multiplicity in current hemisphere.

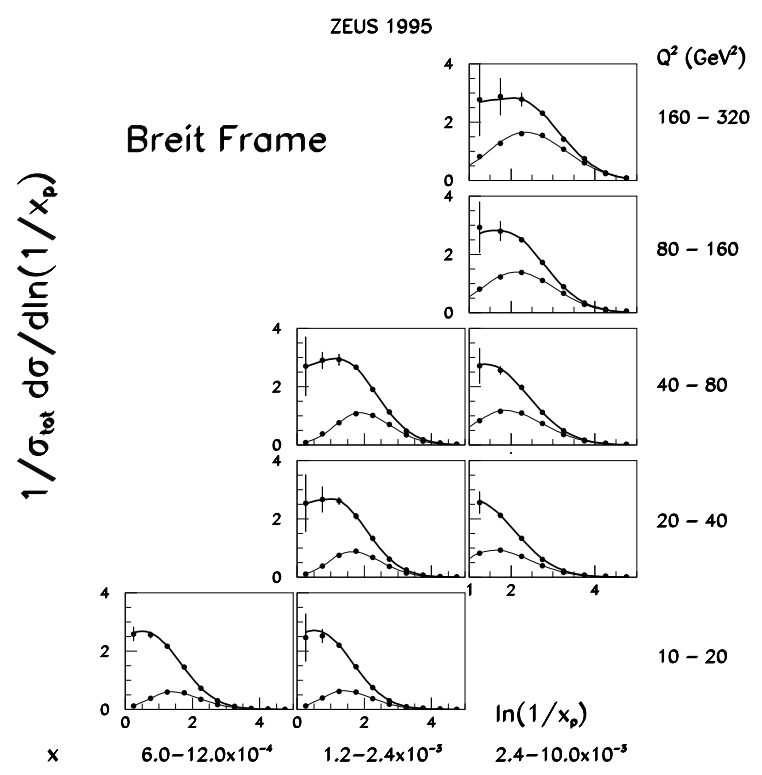

Figure 22: Fragmentation in DIS. Upper data (heavy curve) target region, lower data (light curve) current region. 


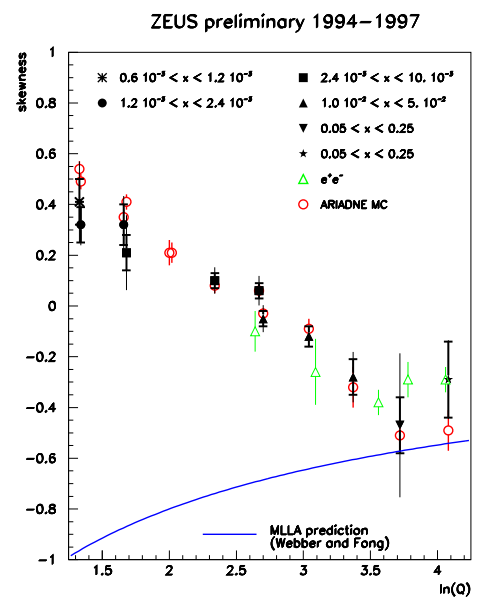

Figure 23: Skewness in current fragmentation region.

The quantity plotted is

$$
\text { Skewness } \equiv\left\langle(\xi-\bar{\xi})^{3}\right\rangle /\left\langle(\xi-\bar{\xi})^{2}\right\rangle^{\frac{3}{2}}
$$

On the other hand, the data lie well below the fixed-order perturbative prediction 58 at low $x_{p}$ and $Q^{2}$ (fig. 24). Discrepancies could be due to power-suppressed $\left(1 / Q^{2}\right)$ corrections, of dynamical and/or kinematical origin. The bands in fig. 24 correspond to an ad-hoc correction factor

$$
\left[1+\left(\frac{m_{\mathrm{eff}}}{Q x_{p}}\right)^{2}\right]^{-1} \quad\left(0.1<m_{\mathrm{eff}}<1 \mathrm{GeV}\right) .
$$

- In the target hemisphere there is also disagreement with MLLA [57, possibly due to the T3 contribution "leaking" into the region $x_{p}<1$. If anything, Monte Carlo models predict too much leakage (fig. 25). Little $Q^{2}$ dependence is evident.

\section{Heavy quark fragmentation}

New data on $b \rightarrow$ B fragmentation from SLD [59], using high-precision vertexing, discriminate between parton-shower plus hadronization models (fig. 26). Note that the data have not yet been corrected for detector effects.

In general one expects the $b$ quark to lose energy by gluon emission in the parton shower, and then to suffer a further non-perturbative energy loss during hadronization. The latter is conventionally parametrized by convoluting with the Peterson function [60]:

$$
f(z)=\frac{1}{z}\left(1-\frac{1}{z}-\frac{\epsilon_{b}}{1-z}\right)^{-2} \quad\left(z=x_{B} / x_{b}\right)
$$




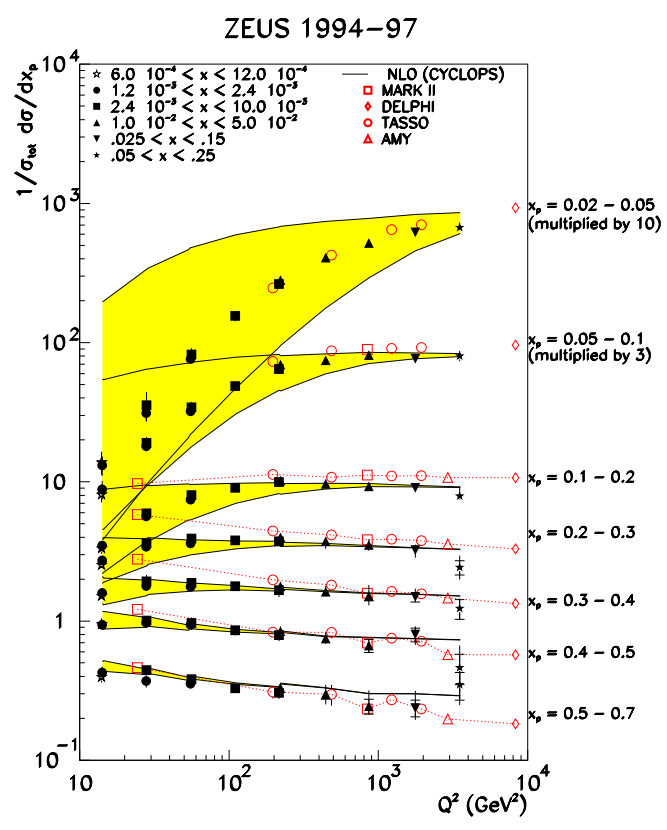

Figure 24: Scaling violation in DIS fragmentation.

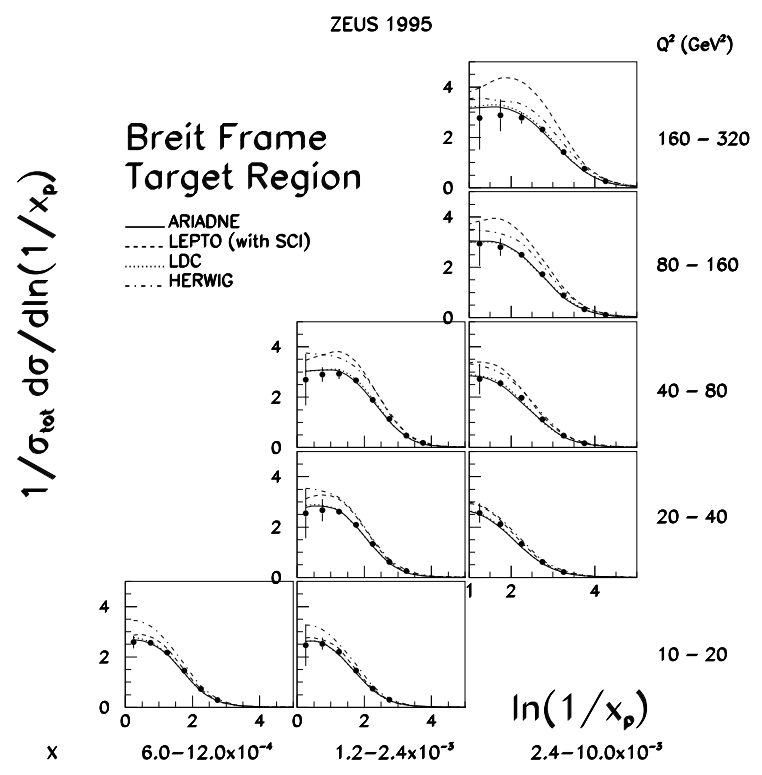

Figure 25: Target fragmentation compared with models. 


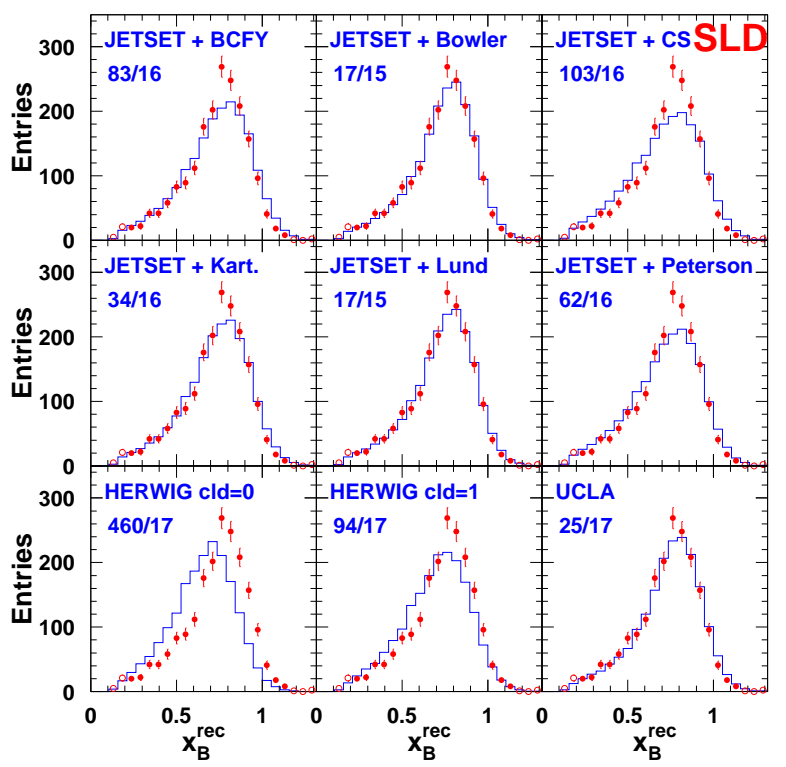

Figure 26: SLD data on $b \rightarrow \mathrm{B}$ fragmentation compared with models.

Including more perturbative QCD leads to a reduction in the amount of non-perturbative smearing required to fit the data, and hence to a smaller fitted value of the Peterson parameter $\epsilon_{b}$ :

Pure Peterson [59]: $\epsilon_{b}=0.036$.

JETSET parton shower + Peterson [59]: $\epsilon_{b}=0.006$.

NLLA QCD + Peterson [61]: $\epsilon_{b}=0.002$.

Here NLLA (next-to-leading logarithmic approximation) refers to an analytical perturbative calculation that goes beyond the parton shower approximation. The calculation of ref. [61] was fitted to earlier ALEPH data 62], as shown in fig. 27.

In the universal low-scale $\alpha_{\mathrm{S}}$ model, the perturbative prediction is extrapolated smoothly to the non-perturbative region, with no Peterson function at all [24].

\section{Conclusions}

We have seen that experimental studies of fragmentation are yielding large amounts of new data for comparison with theoretical predictions and models. Especially impressive is the success of the MLLA perturbative predictions in accounting for the general shape and energy-dependence of fragmentation at small momentum fractions. We are now at the stage when more detailed comparisons await new theoretical input, in the form of coefficient functions that take account of selection procedures, especially for gluon jets in $e^{+} e^{-}$final states.

Comparisons between data and hadronization models suggest that particle masses, rather than quantum numbers, are the dominant factor in suppressing heavy particle production. Baryon production is not yet well described by any model. 


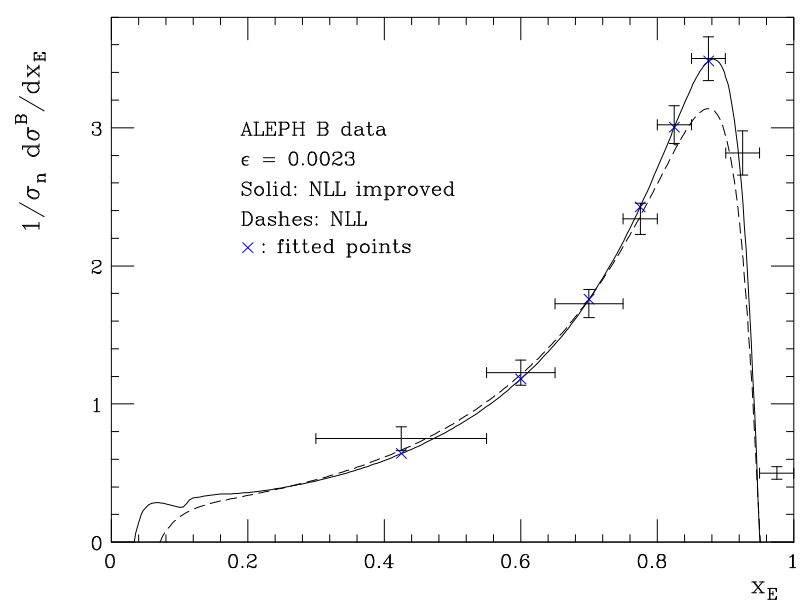

Figure 27: ALEPH data on $b \rightarrow$ B fragmentation compared with NLLA QCD.

Quark and gluon jets have the expected differences and these can be used to measure the ratio of colour factors $C_{A} / C_{F}$. There is no strong evidence yet for different particle content in gluon jets.

Fragmentation in deep inelastic lepton scattering shows some disagreements with perturbative predictions. It is not yet clear whether these are due to higher-order or nonperturbative effects.

New precise $b$ quark fragmentation data from $\mathrm{Z}^{0}$ decay are now available and put strong constraints on models for heavy quark hadronization. The data suggest that perturbative effects dominate.

\section{Acknowledgements}

It is a pleasure to congratulate the organisers for arranging such a successful and truly international Summer School, and to thank the students for making it so lively.

\section{References}

[1] R.K. Ellis, W.J. Stirling and B.R. Webber, "QCD and collider physics," Cambridge, UK: Univ. Pr. (1996) 435 p. (Cambridge monographs on particle physics, nuclear physics and cosmology: 8).

[2] EPS-HEP99 Conference, http://www.physics.helsinki.fi/hep99//

[3] Aleph Collaboration, http://alephwww.cern.ch/ALPUB/conf/conf.htm]

[4] DELPHI Collaboration, http://delphiwww.cern.ch/〜pubxx/delwww/www/delsec/ conferences/tampere99/

[5] L3 Collaboration, http://13www.cern.ch/conferences/EPS99/ 
[6] OPAL Collaboration, http://www.cern.ch/0pal/pubs/eps99_sub.htm]

[7] SLD Collaboration, http://www-pnp.physics.ox.ac.uk/ ${ }^{\text {burrows/tampere/ }}$

[8] H1 Collaboration, http://wWw-h1.desy.de/h1/www/publications/conf/list.tampere99.html

[9] ZEUS Collaboration, http://zedy00.desy.de/conferences99/

[10] D $\emptyset$ Collaboration, http://www-d0.fnal.gov/〜ellison/eps99/eps99.htm]

[11] J.C. Collins and D.E. Soper, Ann. Rev. Nucl. Part. Sci. 37, 383 (1987).

[12] See the fragmentation web pages of the European network "Hadronic Physics with High Energy Electromagnetic Probes" (HaPHEEP) for much useful information on this topic: http://droide1.pv.infn.it/FFdatabase/fragmentation.htm]

[13] Y.L. Dokshitzer, Sov. Phys. JETP 46, 641 (1977).

[14] V.N. Gribov and L.N. Lipatov, Yad. Fiz. 15, 1218 (1972).

[15] G. Altarelli and G. Parisi, Nucl. Phys. B126, 298 (1977).

[16] B.I. Ermolaev and V.S. Fadin, JETP Lett. 33, 269 (1981).

[17] A.H. Mueller, Phys. Lett. 104B, 161 (1981).

[18] S.J. Brodsky and J.F. Gunion, Phys. Rev. Lett. 37, 402 (1976).

[19] Y.I. Azimov, Y.L. Dokshitzer, V.A. Khoze and S.I. Troian, Zeit. Phys. C31, 213 (1986).

[20] C.P. Fong and B.R. Webber, Phys. Lett. B229, 289 (1989); Nucl. Phys. B355, 54 (1991).

[21] Y.L. Dokshitzer, V.A. Khoze, A.H. Mueller and S.I. Troian, "Basics of perturbative QCD," Gif-sur-Yvette, France: Ed. Frontieres (1991) 274 p. (Basics of).

[22] Y.I. Azimov, Y.L. Dokshitzer, V.A. Khoze and S.I. Troian, Z. Phys. C27, 65 (1985).

[23] Y.L. Dokshitzer and B.R. Webber, Phys. Lett. B352, 451 (1995) hep-ph/9504219.

[24] Y.L. Dokshitzer, V.A. Khoze and S.I. Troian, Phys. Rev. D53, 89 (1996) hepph/9506425.

[25] Y.L. Dokshitzer, G. Marchesini and B.R. Webber, Nucl. Phys. B469, 93 (1996) hepph/9512336.

[26] G. Marchesini and B.R. Webber, Nucl. Phys. B238, 1 (1984).

[27] B.R. Webber, Nucl. Phys. B238, 492 (1984).

[28] G. Marchesini and B.R. Webber, Nucl. Phys. B310, 461 (1988). 
[29] G. Marchesini, B.R. Webber, G. Abbiendi, I.G. Knowles, M.H. Seymour and L. Stanco, Comput. Phys. Commun. 67, 465 (1992); hep-ph/9607393.

[30] D. Amati and G. Veneziano, Phys. Lett. 83B, 87 (1979).

[31] G. Marchesini, L. Trentadue and G. Veneziano, Nucl. Phys. B181, 335 (1981).

[32] I.G. Knowles and G.D. Lafferty, J. Phys. G G23, 731 (1997) hep-ph/9705217.

[33] B. Andersson, G. Gustafson, G. Ingelman and T. Sjostrand, Phys. Rept. 97, 31 (1983).

[34] T. Sjostrand, Comput. Phys. Commun. 39, 347 (1986).

[35] T. Sjostrand and M. Bengtsson, Comput. Phys. Commun. 43, 367 (1987).

[36] T. Sjostrand, Comput. Phys. Commun. 82, 74 (1994); CERN-TH-7112-93, hepph/9508391.

[37] C.D. Buchanan and S.B. Chun, Phys. Rev. Lett. 59, 1997 (1987).

[38] S.B. Chun and C.D. Buchanan, Phys. Lett. B308, 153 (1993); Phys. Rept. 292, 239 (1998).

[39] G. Rudolph, private communication.

[40] P.V. Chliapnikov, Phys. Lett. B462, 341 (1999).

[41] G. Alexander et al. [OPAL Collaboration], Z. Phys. C73, 569 (1997).

[42] DELPHI Collaboration, EPS-HEP99 paper 3_147.

[43] DELPHI Collaboration, EPS-HEP99 paper 1_225.

[44] DELPHI Collaboration, EPS-HEP99 paper 1_225.

[45] CDF Collaboration, EPS-HEP99 paper 1_600.

[46] K. Abe et al. [SLD Collaboration], hep-ex/9908033.

[47] DELPHI Collaboration, EPS-HEP99 paper 3_146.

[48] OPAL Collaboration, EPS-HEP99 paper 1_4.

[49] G. Abbiendi et al. [OPAL Collaboration], Eur. Phys. J. C11, 217 (1999) hepex/9903027.

[50] DELPHI Collaboration, EPS-HEP99 paper 1_571.

[51] S. Catani, Y.L. Dokshitzer, M. Olsson, G. Turnock and B.R. Webber, Phys. Lett. B269, 432 (1991).

[52] S. Catani, B.R. Webber, Y.L. Dokshitzer and F. Fiorani, Nucl. Phys. B383, 419 (1992).

[53] P. Eden, G. Gustafson and V. Khoze, Eur. Phys. J. C11, 345 (1999) hep-ph/9904455. 
[54] P. Abreu et al. [DELPHI Collaboration], Phys. Lett. B449, 383 (1999) hepex/9903073.

[55] DELPHI Collaboration, private communication.

[56] C. Adloff et al. [H1 Collaboration], Nucl. Phys. B504 (1997) 3 hep-ex/9707005.

[57] J. Breitweg et al. [ZEUS Collaboration], Eur. Phys. J. C11, 251 (1999) hepex/9903056.

[58] D. Graudenz, Phys. Lett. B406, 178 (1997) hep-ph/9606470.

[59] K. Abe et al. [SLD Collaboration], hep-ex/9908032.

[60] C. Peterson, D. Schlatter, I. Schmitt and P. Zerwas, Phys. Rev. D27, 105 (1983).

[61] P. Nason and C. Oleari, hep-ph/9903541.

[62] D. Buskulic et al. [ALEPH Collaboration], Phys. Lett. B357, 699 (1995). 